\title{
The pelvis: "Strange attractor" of the postural system. A study based on a group of 332 children between 2 and 12 years old
}

\section{A Fimiani}

Address: via Dello Stadio 79 Ischia (NA) - 80077, Italy

Email: A Fimiani - fimianiantonio@libero.it

from 6th International Conference on Conservative Management of Spinal Deformities Lyon, France. 21-23 May 2009

Published: 14 December 2009

Scoliosis 2009, 4(Suppl 2):P4 doi: I0.I| 86/| 748-7|6|-4-S2-P4

This abstract is available from: http://www.scoliosisjournal.com/content/4/S2/P4

(C) 2009 Fimiani; licensee BioMed Central Ltd.

\section{Objectives}

The aim of this work is to show that the pelvis represents the focal point of the postural system, or rather, the link between muscular and skeletal systems. The pelvis is the seat of insertion of lower extremity and trunk musculature, and these muscles are controlled by the postural system. The afferent postural information relayed by this musculature conditions the spatial order of the musculoskeletal system, so that correct afferent conditioning leads to proper musculoskeletal orientation aligned with the center of gravity.

\section{Background}

Theories about postural systems were born during the 1970 's, and they are mainly represented by the Fine Postural System by P. M. Gagey and the Tonic Postural System by B. Bricot. Moreover, in 2005 J. C. de Mauroy hypothesized the existence of a "strange attractor" that provides a foundation for the vertebral curvature of a child's spine. Ontogenesis has shown that the pelvis takes on the role of body stabilizer in the space as the human body becomes vertically oriented.

\section{Materials and methods}

During 5 years, 332 children were observed, ages 2 to 12 years old. In this population, 210 children started receptorial therapy for a period of 24 months. 182 patients showed at the first visit an AP and lateral full-spine $\mathrm{x}$-ray. During the first visit and at three months intervals, children were imaged in the frontal (anterior, posterior) and sagittal planes, in standard position with plantar support with an angle of 30 degrees.

\section{Outcome}

Clinical evidence shows that in 12 months, the pelvis realigns, with a subsequent re-established balance of the legs. In 33 patients with scoliosis and dorsolumbar deviation (Cobb angle between 10 and 18 degrees), a significant improvement was shown at 12 months $(\mathrm{p}=0.0005 ; \mathrm{r}=$ $0.708)$. In 29 patients with a Risser score of zero and dorsolumbar deviation (Cobb angle between 10 and 30 degrees and vertebral rotation between 5 and 15 degrees), a significant improvement of curves was shown at 24 months.

\section{Conclusion}

The pelvis is the focal point, the "strange attractor," of the musculoskeletal system, it is managed by the automatic postural system, and an automatic postural component exists even in idiopathic scoliosis. 